\title{
Barreiras para a prática de atividade física e estágio de mudança de comportamento em servidores de um hospital escola do sul do Brasil
}

\author{
Barriers to the practice of physical activity and stage of behavior change in employees of a teaching \\ hospital in southern Brazil \\ Barreras para la práctica de actividad física y etapa de cambio de comportamiento en empleados \\ de un hospital universitario en el sur de Brasil
}

Recebido: 19/08/2021 | Revisado: 26/08/2021 | Aceito: 21/09/2021 | Publicado: 22/09/2021

\author{
Ana Silvia Degasperi Ieker \\ ORCID: https://orcid.org/0000-0001-9863-9598 \\ Universidade Estadual de Maringá, Brasil \\ E-mail: anasilviaieker@hotmail.com \\ Rui Gonçalves Marques Elias \\ ORCID: https://orcid.org/0000-0002-0040-8212 \\ Universidade Estadual do Norte do Paraná, Brasil \\ E-mail: rgmelias@uenp.edu.br \\ Fernanda Marinho \\ ORCID: https://orcid.org/0000-0001-5845-5196 \\ Universidade Estadual de Maringá, Brasil \\ E-mail: fer-nanda1908@live.com \\ Daniel Vicentini de Oliveira \\ ORCID: https://orcid.org/0000-0002-0272-9773 \\ Universidade Cesumar, Brasil \\ E-mail: d.vicentini@hotmail.com \\ José Roberto Andrade do Nascimento Júnior \\ ORCID: https://orcid.org/0000-0003-3836-6967 \\ Universidade Federal do Vale do São Francisco, Brasil \\ E-mail: jroberto.jrs01@gmail.com \\ Fábio Ricardo Acêncio \\ ORCID: https://orcid.org/0000-0002-6389-6196 \\ Universidade Cesumar, Brasil \\ E-mail: fabioacencio@hotmail.com \\ Rose Mari Bennemann \\ ORCID: https://orcid.org/0000-0003-3946-6396 \\ Universidade Cesumar, Brasil \\ E-mail: rose.bennemann@unicesumar.edu.br \\ Ana Luiza Barbosa Anversa \\ ORCID: https://orcid.org/0000-0003-4363-3433 \\ Universidade Estadual de Maringá, Brasil \\ E-mail: ana.beah@gmail.com \\ Wilson Rinaldi \\ ORCID: https://orcid.org/0000-0001-5593-3666 \\ Universidade Estadual de Maringá, Brasil \\ E-mail: wrinaldi@uem.br
}

\begin{abstract}
Resumo
Este estudo teve o objetivo de analisar a associação das barreiras auto referidas para a prática de atividade física e estágio de mudança de comportamento em servidores de um Hospital Escola do Sul do Brasil. Estudo transversal, o qual investigou 485 servidores de um Hospital Escola do Sul do Brasil, sendo 313 mulheres e 172 homens. As variáveis analisadas foram: nível de atividade física, índice de massa corporal, circunferência abdominal, idade, tabagismo, consumo de álcool, horas despendidas de sono, estágio de mudança de comportamento e barreiras para a prática de atividade física. A estatística descritiva foi utilizada em valores de mediana, mínimo e máximo para caracterização da população, além da utilização do teste U de Mann-Whitney para comparação entre os sexos. Foi utilizado o teste Quiquadrado $(\mathrm{p}<0,05)$ com correção de Fisher, quando necessário. No sexo masculino, as barreiras: não gostar de atividade física $(p=0,018)$, medo de lesão $(p=0,003)$ e falta de companhia $(p=0,005)$ demonstraram diferença significativa entre comportamentos inativos e ativos. As barreiras; falta de tempo livre, medo de lesão e preguiça demonstraram uma diferença significativa entre as faixas etárias, não demonstrando progressão no decorrer do tempo. O baixo nível de
\end{abstract}


atividade física pode ser considerado um fator associado a maiores queixas de limitações para a prática de atividade física.

Palavras-chave: Saúde do trabalhador; Barreiras; Atividade física; Adultos.

\begin{abstract}
This study aimed to analyze the association of self-reported barriers to the practice of physical activity and stage of behavior change in employees of a Teaching Hospital in southern Brazil. Cross-sectional study, which investigated 485 workers at a Teaching Hospital in southern Brazil, 313 women and 172 men. The variables analyzed were: level of physical activity, body mass index, waist circumference, age, smoking, alcohol consumption, hours spent sleeping, stage of behavior change and barriers to physical activity. Descriptive statistics were used in median, minimum and maximum values to characterize the population, in addition to using the Mann-Whitney $U$ test for comparison between genders. The Chi-square test $(\mathrm{p}<0.05)$ with Fisher correction was used, when necessary. In males, the barriers: not liking physical activity $(\mathrm{p}=0.018)$, fear of injury $(\mathrm{p}=0.003)$ and lack of company $(\mathrm{p}=0.005)$ showed a significant difference between inactive and active behaviors. The barriers; lack of free time, fear of injury and laziness showed a significant difference between age groups, not showing progression over time. The low level of physical activity can be considered a factor associated with greater complaints of limitations in the practice of physical activity.
\end{abstract}

Keywords: Motor activity; Cognition; Depression; Exercise.

\title{
Resumen
}

Este estudio tuvo como objetivo analizar la asociación de barreras autoinformadas para la práctica de actividad física y etapa de cambio de conducta en empleados de un Hospital Docente del sur de Brasil. Estudio transversal, que investigó a 485 trabajadores de un Hospital Docente del sur de Brasil, 313 mujeres y 172 hombres. Las variables analizadas fueron: nivel de actividad física, índice de masa corporal, perímetro de cintura, edad, tabaquismo, consumo de alcohol, horas de sueño, etapa de cambio de conducta y barreras a la actividad física. Se utilizó estadística descriptiva en valores medianos, mínimos y máximos para caracterizar la población, además de utilizar la prueba U de Mann-Whitney para la comparación entre géneros. Se utilizó la prueba de Chi-cuadrado $(\mathrm{p}<0.05)$ con corrección de Fisher, cuando fue necesario. En los hombres, las barreras: no gustarle la actividad física $(p=0,018)$, miedo a lesionarse $(p=0,003)$ y falta de compañía $(\mathrm{p}=0,005)$ mostraron una diferencia significativa entre las conductas inactivas y activas. Las barreras; La falta de tiempo libre, el miedo a lesionarse y la pereza mostraron una diferencia significativa entre los grupos de edad, no mostrando progresión en el tiempo. El bajo nivel de actividad física puede considerarse un factor asociado a mayores quejas de limitaciones en la práctica de actividad física.

Palabras clave: Actividad motora; Cognición; Depresión; Ejercicio.

\section{Introdução}

A prática de atividade física $(\mathrm{AF})$, reconhecida na maioria das vezes, como um tratamento não farmacológico, acarreta mudanças fisiológicas e psicossociais (Alves \& Pontelli, 2015) que auxiliam na prevenção e tratamento de determinadas doenças, principalmente nas doenças crônicas. Aliado a isso, essa prática auxilia a melhoria de qualidade de vida dos praticantes, diminuição de filas de espera em serviços de atenção básica, redução do número de internações por doenças recorrentes a esta causa, que afetam diretamente no custo da saúde (Bittencourt, Santos, \& Oliveira, 2012).

Dados da Pesquisa Nacional de Saúde (PNS) estimou a prevalência de adultos insuficientemente ativos no Brasil de 46,0\% (IC95\% 45,2\%;46,8\%) em 2013 (Berria, Teixeira, Minatto, \& Petrosli, 2016). A adesão de um baixo nível populacional, pode ser explicada pela dificuldade de mudança de hábitos inativos constituídos ao longo da vida (Éric \& Almeida, 2012) além da existência de diversas barreiras que prejudicam esse processo de manutenção.

Em relação aos trabalhadores, os baixos níveis de AF, podem predispor distúrbios ocupacionais, como lesões por esforço repetitivo e distúrbios musculoesqueléticos relacionados ao trabalho (Frank, Dresner, Shani, \& Vinker, 2013), além de interferir em aspectos motivacionais, nota-se também que demandas exaustivas de trabalho é outro fator que influencia para esta prática no lazer (Franklim C, Daniele, Vitorino, Cieslak, \& Gregorio, 2008). Esta inatividade física e hábitos não saudáveis, consequentemente, impactam na redução da participação na força de trabalho, no quantitativo de horas trabalhadas, na maior rotatividade de empregos, nas aposentadorias precoces, assim como no comprometimento dos salários, ganhos e posição alcançada (Fransson, Heikkilä, Nyberg, Zins, Westerlund, Westerholm, \& Väänänen, 2012).

Mesmo com características particulares, o local de trabalho é destaque para promoção da saúde, logo se faz necessário 
estratégias de implantação de uma cultura preventiva contra os fatores de risco com ações efetivas e de baixo custo (Goulart, 2011). Além de que, o engajamento de profissionais de saúde a comportamentos saudáveis, aumentam a propensão do aconselhamento preventivo aos seus pacientes (Hyeda, Costa, Sbardellotto, Ferreira, 2015).

Nesse sentido, se faz necessário conhecer os limites que afastam os trabalhadores da atividade física e ressaltar a importância de um estilo de vida ativo no decorrer da vida. Sendo assim, o objetivo desse trabalho foi analisar a associação das barreiras auto referidas para a prática de atividade física e estágio de mudança de comportamento em servidores de um Hospital Escola do Sul do Brasil.

\section{Metodologia}

Este é um estudo quantitativo, com delineamento transversal e observacional, aprovado pelo Comitê de Ética em Pesquisa, por meio do parecer número 1.766.685.

\subsection{Participantes}

O número total de servidores foi levantado a partir de uma listagem fornecida pela Divisão de Recursos Humanos do Hospital, totalizando 701 servidores efetivos, distribuídos em uma Superintendência e quatro diretorias: Diretoria Administrativa-DAI ( $n=261$ ); Diretoria de Enfermagem-DEE ( $n=296$ ); Diretoria Farmacêutica/ Laboratorial-DAF ( $n=76)$ e Diretoria do Hemocentro-DHE ( $\mathrm{n}=68)$.

\subsection{Instrumentos}

Dados sociodemográficos. Para avaliar o perfil sociodemográfico dos participantes, um questionário semiestruturado foi desenvolvido pelos autores com questões sobre sexo (homens, mulheres), idade, faixa etária (menos de 40 anos; 40 a 50 anos; mais de 50 anos, tempo de contratação (anos), escolaridade (anos de estudo) e jornada semanal (horas)

Nível de atividade física. A Atividade física habitual foi analisada a partir das respostas ao Questionário Internacional de Atividade Física (IPAQ-8 versão reduzida) validado para o Brasil. Sendo computada, em forma de entrevista, a frequência, duração e intensidade das atividades físicas realizadas na semana anterior a coleta de dados. O questionário classifica como ativos fisicamente aqueles que realizam 150 minutos por semana de atividades físicas de intensidade moderada a vigorosa; irregularmente ativo: quem atinge entre 10 a 149 minutos por semana de atividades físicas de intensidade moderada a vigorosa; sedentária: indivíduo que não atinge o mínimo de 10 minutos por semana de atividades físicas de intensidade moderada a vigorosa.

Dados antropométricos. A avaliação da massa corporal foi realizada por meio de uma balança digital Welmy, com precisão de 100 gramas e a estatura através de um estadiômetro portátil (Welmy®), com precisão de 0,1 centímetros. Todos os indivíduos foram orientados a estarem descalços, em posição ereta, pés juntos e braços posicionados ao longo do corpo. O índice de massa corporal (IMC) foi calculado através da divisão da massa corporal em quilogramas pela estatura em metros elevada ao quadrado. O perímetro abdominal foi aferido no ponto umbilical, entre a última costela flutuante e a crista ilíaca mediante uma fita antropométrica inextensível (Sanny®).

O Estágio de Mudança de Comportamento foi classificado através do questionário proposto por Marcus, Banspach, Lefebvre, Rossi, Carleton, \& Abrams, 1992), dividido em cinco estágios: 1) Pré-contemplação (indivíduo não pretende mudar o comportamento); 2) Contemplação (há intenção de mudar de comportamento, mas não imediatamente); 3) Preparação (indivíduo não está envolvido, mas pretende fazê-lo dentro dos próximos 30 dias); 4) Ação (indivíduo está regularmente ativo por menos de seis meses), e 5) Manutenção (indivíduo é ativo a mais de seis meses). A partir da resposta do EMC, os servidores foram classificados em um estado de comportamento "inativo", estágios 1, 2 e 3, e "ativo", estágios 4 e 5. 
Foi aplicado o questionário de percepção de barreiras pessoais para a prática de atividade física. No presente estudo, o questionário foi adaptado, composto somente por respostas fechadas (sim ou não) e análise de oito barreiras (possuir alguma lesão ou doença, gostar de praticar atividade física, sentir preguiça ou cansaço, falta de dinheiro, medo de se machucar, falta de companhia, falta de espaço público e falta de tempo livre).

\subsection{Procedimentos de Coleta dos Dados}

Após a aprovação do Comitê de Ética, a coleta procedeu: 1) divulgação e autorização com a superintendência e gerências de cada diretoria; 2) definição e estratificação da logística com a chefia de cada setor; 3) entrada e definição do melhor horário nos setores; 4) divulgação da pesquisa e entrega do TCLE para cada servidor; 5) realização da entrevista e avaliação física.

As avaliações foram realizadas logo após a entrevista, contando com medidas diretas de peso e estatura, aferição da pressão arterial e circunferência abdominal. A coleta de dados foi realizada no próprio local de trabalho do servidor, sendo que as entrevistas ocorreram diariamente nos três períodos (manhã, tarde e noite). Os dados foram coletados por uma equipe de cinco entrevistadores familiarizados, treinados para aplicação dos instrumentos de pesquisa de forma padrão.

\subsection{Análise de Dados}

A análise de dados foi realizada através de estatísticas descritivas e inferenciais. Inicialmente, o banco de dados foi verificado e potenciais erros foram corrigidos. A estatística descritiva foi utilizada em valores de mediana, mínimo e máximo para caracterização da população. Para comparar as médias entre dois grupos, utilizou-se o teste U de Mann-Whitney para amostras independentes não-paramétricas.

Para a associação dos dados, foi utilizado o teste Qui-quadrado com correção de Fisher, quando necessário. Com o intuito de ajustar as variáveis conforme os objetivos do estudo, foi realizada uma análise Qui-quadrado ajustada, por sexo e faixa etária. Todas as análises foram realizadas por meio do Statistical Package for Social Science (SPSS), versão 20.0.

\section{Resultados}

Dos 485 servidores participantes da pesquisa 35,5\% (172) eram do sexo masculino e 64,5\% (313) do sexo feminino. Os dados descritivos foram expressos na forma de tendência central (mediana) e medidas de disperção (valores mínimos e máximos). A Tabela 01 evidencia a característica da população com os dados antropométricos (peso, estatura, IMC e Circunferência da Cintura) medida pressórica e informações laborais.

A variável estatura $(p<0,001)$, massa corporal $p<(0,001)$, circunferência abdominal $(p<0,001)$, pressão arterial sistólica e diastólica $(\mathrm{p}<0,001)$ demonstraram uma diferença significativa entre os sexos, além de diferenças no quantitativo de horas semanais de trabalho $(\mathrm{p}=0,004)$ e horas de sono $(\mathrm{p}=0,003)$, conforme descritos na tabela abaixo. 
Tabela 1. Idade, características antropométricas, hemodinâmicas, trabalho, atividade física e horas de sono dos servidores de um Hospital Escola do Sul do Brasil, 2017 (n=485).

\begin{tabular}{lccc}
\hline \multirow{2}{*}{ Variáveis } & \multicolumn{2}{c}{ Mediana (mín - máx) } & \multirow{2}{*}{ (valor) } \\
\cline { 2 - 3 } & $\begin{array}{c}\text { HOMENS } \\
(\mathbf{n}=\mathbf{1 7 2})\end{array}$ & $\begin{array}{c}\text { MUHERES } \\
(\mathbf{n}=\mathbf{3 1 3})\end{array}$ & 0,055 \\
\hline Idade (anos) & $47,3(23,8-66,9)$ & $48,0(22,3-65,9)$ & $<0,001^{*}$ \\
Massa (kg) & $80,6(51,1-138,3)$ & $70(41,2-135,0)$ & $<0,001^{*}$ \\
Estatura (cm) & $1,70(1,5-1,9)$ & $1,60(1,5-1,9)$ & 0,240 \\
IMC (kg/m $)$ & $27,62(18,7-45,7)$ & $27,12(18,56-46,7)$ & $<0,001^{*}$ \\
CA (cm) & $95(69-130)$ & $90(67-128)$ & 0,829 \\
Tempo de contratação (anos) & $20(1-39)$ & $20(1-42)$ & 0,188 \\
Escolaridade (anos de estudo) & $15(4-15)$ & $15(4-15)$ & $0,004^{*}$ \\
Jornada semanal (horas) & $40(12-80)$ & $40(24-80)$ & \\
\hline
\end{tabular}

Teste U de Mann - Whitney; *Valores significativos $\mathrm{p} \leq 0,05$.

IMC: Índice de Massa Corporal; CA: Circunferência Abdominal.

Fonte: os autores.

A avaliação das barreiras percebidas para a prática de atividade física indicou as variáveis preguiça $(44,1 \%)$, falta de tempo livre $(63,1 \%)$ e falta de companhia $(34,1 \%)$ como principais motivos que impedem ou dificultam os indivíduos se engajarem na prática de atividades físicas (Tabela 2). Porém, as barreiras: falta de companhia, tempo livre e falta de espaço público demonstraram diferenças significativas entre os sexos.

Tabela 2. Identificação das barreiras auto referidas para a prática de atividade física entre os servidores de um Hospital Escola do Sul do Brasil, 2017 ( $\mathrm{n}=485)$.

\begin{tabular}{lcccc}
\hline \multirow{2}{*}{ Motivo } & \multicolumn{4}{c}{ Barreiras percebidas } \\
\cline { 2 - 4 } & $\mathbf{H}$ & $\mathbf{M}$ & Total & p (valor) \\
\hline Não gostar de AF & $\mathbf{( 1 7 2 )}$ & $\mathbf{( 3 1 3 )}$ & $\mathbf{( 4 8 5 )}$ & 0,079 \\
Preguiça & $27(15,7)$ & $70(22,4)$ & $214(44,1)$ & 0,089 \\
Dinheiro & $67(39,0)$ & $147(47,0)$ & $103(21,2)$ & 0,293 \\
Lesão & $32(18,6)$ & $71(22,7)$ & $110(22,7)$ & 0,652 \\
Segurança & $41(23,8)$ & $69(22,0)$ & $87(17,9)$ & 0,436 \\
Companhia & $34(19,8)$ & $53(16,9)$ & $167(34,4)$ & $0,041^{*}$ \\
Tempo livre & $49(28,5)$ & $118(37,7)$ & $306(63,1)$ & $0,014^{*}$ \\
Espaço Público & $96(55,8)$ & $210(67,1)$ & $82(16,9)$ & $0,045^{*}$ \\
\hline
\end{tabular}

$*$ Valores significativos $\mathrm{p} \leq 0,05$.

AF: atividade física.

Fonte: os autores.

A Tabela 3 apresenta as barreiras auto referidas para a prática de atividade física de acordo com o EMC (comportamentos ativos e inativos). Os homens deste estudo apresentaram diferença significativa entre os comportamentos 
inativos e ativos nas barreiras: não gostar de $\mathrm{AF}(\mathrm{p}=0,018)$, lesão $(\mathrm{p}=0,003)$ e companhia $(\mathrm{p}=0,005)$. Já entre as mulheres, o tempo livre, a preguiça e a falta de companhia são as principais barreiras, apresentando diferença significativa entre os comportamentos, somente na barreira medo de lesão $(\mathrm{p}=0,000)$. Analisando o total de barreiras, percebe-se que os homens do comportamento inativo apresentam mais barreiras, quando comparadas aos outros grupos.

Tabela 3. Associação entre o Estágio de Mudança de Comportamento e Barreiras auto referidas para a prática de atividade física de servidores de um Hospital do Sul do Brasil, 2016 (n=485).

\begin{tabular}{lcccccccc}
\hline \multirow{2}{*}{ Barreiras } & \multicolumn{7}{c}{ Homens } & \multicolumn{7}{c}{ Mulheres } \\
\cline { 2 - 9 } & $\begin{array}{c}\text { Comp. Ativo } \\
(\mathbf{n = 6 7 )}\end{array}$ & $\begin{array}{c}\text { Comp. Inativo } \\
(\mathbf{n = 1 0 5})\end{array}$ & $\begin{array}{c}\text { Total } \\
\mathbf{N}(\boldsymbol{\%})\end{array}$ & $\mathbf{p}$ & $\begin{array}{c}\text { Comp. Ativo } \\
(\mathbf{n = 1 0 3})\end{array}$ & $\begin{array}{c}\text { Comp. Inativo } \\
(\mathbf{n = 2 0 9})\end{array}$ & Total & $\mathbf{p}$ \\
& $\mathbf{N}(\boldsymbol{\%})$ & $\mathbf{N}(\boldsymbol{\%})$ & & & $\mathbf{N}(\boldsymbol{\%})$ & $\mathbf{N}(\boldsymbol{\%})$ & $\mathbf{N}(\boldsymbol{\%})$ & \\
\hline Não gostar de AF & $5(18,5)$ & $22(81,5)$ & $27(15,7)$ & $0,018^{*}$ & $17(16,5)$ & $53(25,4)$ & $70(22,4)$ & 0,078 \\
Preguiça & $26(38,8)$ & $41(39,0)$ & $67(39,0)$ & 0,975 & $44(42,7)$ & $102(48,8)$ & $147(47,0)$ & 0,313 \\
Lesão & $8(11,9)$ & $33(31,4)$ & $41(23,8)$ & $0,003^{*}$ & $35(34,0)$ & $34(16,3)$ & $69(22,0)$ & $<0,001^{*}$ \\
Dinheiro & $9(13,4)$ & $23(21,9)$ & $32(18,6)$ & 0,164 & $19(18,4)$ & $52(24,9)$ & $71(22,7)$ & 0,204 \\
Segurança & $13(19,4)$ & $21(20,0)$ & $34(19,8)$ & 0,924 & $18(17,5)$ & $35(16,7)$ & $53(16,9)$ & 0,872 \\
Companhia & $11(16,4)$ & $38(36,2)$ & $49(28,5)$ & $0,005^{*}$ & $36(35,0)$ & $82(39,2)$ & $118(37,7)$ & 0,465 \\
Tempo livre & $32(47,8)$ & $64(61,0)$ & $96(55,8)$ & 0,089 & $64(62,1)$ & $145(69,4)$ & $210(67,1)$ & 0,202 \\
Espaço público & $12(17,9)$ & $25(23,8)$ & $37(21,5)$ & 0,359 & $15(14,6)$ & $30(14,4)$ & $45(14,4)$ & 0,961 \\
\hline
\end{tabular}

Teste Qui-Quadrado; *Valores significativos $\mathrm{p} \leq 0,05$.

AF: atividade física.

Fonte: os autores.

A Tabela 4 descreve as barreiras auto referidas associada com a faixa etária, na população geral. As barreiras preguiça, medo de sofrer lesão e falta de tempo livre demonstraram associação significativa com a maior idade, respectivamente $\mathrm{p}=0,007$, $\mathrm{p}=0,036$ e $\mathrm{p}=0,046$.

Tabela 4. Barreiras auto referidas para a prática de atividade física e sua associação com faixa etária de servidores de um Hospital Escola do Sul do Brasil, 2017. $(n=485)$

\begin{tabular}{|c|c|c|c|c|c|}
\hline Barreira & Total $(\%)$ & $\begin{array}{c}\text { Menos de } 40 \text { anos } \\
\text { N (\%) }\end{array}$ & $\begin{array}{c}40 \text { a } 50 \text { anos } \\
\text { N }(\%)\end{array}$ & $\begin{array}{c}\text { Mais de } 50 \text { anos } \\
\text { N (\%) }\end{array}$ & p-valor \\
\hline Não gostar de AF & $97(20,0)$ & $15(14,2)$ & $45(21,2)$ & $37(22,2)$ & 0,229 \\
\hline Preguiça & $214(44,1)$ & $61(57,5)^{\mathrm{a}}$ & $85(40,1)^{a, c}$ & $68^{\mathrm{b}}(40,7)$ & $0,007 *$ \\
\hline Lesão & $110(22,7)$ & $22(20,8)^{\mathrm{a}}$ & $39(18,4)^{b, c}$ & $49(29,3)^{\mathrm{a}, \mathrm{b}}$ & $0,036^{*}$ \\
\hline Dinheiro & $103(21,2)$ & $23(21,7)$ & $44(20,8)$ & $36(21,6)$ & 0,974 \\
\hline Segurança & $87(17,9)$ & $19(17,9)$ & $31(14,6)$ & $37(22,2)$ & 0,165 \\
\hline Companhia & $167(34,4)$ & $31(29,2)$ & $71(33,5)$ & $65(38,9)$ & 0,242 \\
\hline Tempo livre & $306(63,1)$ & $76(71,7)^{\mathrm{a}}$ & $135(63,7)^{\mathrm{a}, \mathrm{c}}$ & $95(56,9)^{\mathrm{a}, \mathrm{b}}$ & $0,046^{*}$ \\
\hline Espaço público & $82(16,9)$ & $20(18,9)$ & $31(14,6)$ & $31(18,6)$ & 0,496 \\
\hline
\end{tabular}

Diferença em relação a faixa etária: a-menos de 40; b-40 a 50 e c- mais de 50.

AF: atividade física.

Fonte: os autores. 


\section{Discussão}

Este estudo teve como objetivo analisar a associação das barreiras auto referidas para a prática de atividade física e estágio de mudança de comportamento em servidores de um Hospital Escola do Sul do Brasil. Os principais resultados revelaram maior proporção de homens inativos que reportaram barreiras como não gostar de AF, lesão e falta de companhia, enquanto que somente a barreira medo de lesão apresentou maior proporção entre as mulheres ativas fisicamente. Além disso, as barreiras preguiça, medo de sofrer lesão e falta de tempo livre demonstraram associação com a faixa etária, entretanto, não se percebeu tendência de progressão conforme o aumento da idade.

Os resultados da presente pesquisa se diferem dos indicados na literatura, que retrata que, entre os homens, as principais barreiras para a prática de atividade física se remetem a compromissos familiares e jornada de trabalho (Jesus \& Jesus, 2011; Abreu, Abreu, Sampaio, \& Rodrigues, 2018).

Temos que destacar que as barreiras para praticar atividade física não possuem relação apenas com o sexo, mas principalmente com a cultura, moradia, condição socioeconômica e hábitos de vida diversos. Não gostar de AF é uma barreira real, e pode sim justificar o motivo dos homens, nessa pesquisa, serem inativos.

Na revisão sistemática realizada por Vieira e da Silva (2019), nota-se que as barreiras recorrentes para a prática de atividade física, sem considerar o sexo e/ou ocupação, são ambiente insuficientes seguros, falta de companhia, limitações e cansaço físico, falta de interesse em praticar e medo de sofrer lesão. Aqui vemos diferentes tipos de barreiras, o que reforça a hipótese levantada no parágrafo anterior, a respeito de diversos motivos impactarem nas barreiras para a prática de AF entre as pessoas.

Encontramos que a barreira medo de lesão apresentou maior proporção entre as mulheres ativas fisicamente. Infere-se que essas mulheres sejam fisicamente ativas, justamente para diminuir o risco de lesões no dia a dia, mas este não foi um dado avaliado na presente pesquisa. Estudo de Gomes, Papini, Nakamura, Teixeira, e Kokobun (2019) encontrou que, as barreiras mais reportadas pelo grupo de mulheres que não realizavam exercícios físicos, a falta de companhia (40,9\%), falta de energia (38,7\%), sentir-se muito cansada ou desmotivada. A barreira falta de tempo é geralmente a mais comum em outras pesquisas, no entanto não se mostrou fortemente presente neste estudo (Gobbi et al., 2012; Justine et al., 2013; Normansell et al., 2014)

As barreiras preguiça, medo de sofrer lesão e falta de tempo livre demonstraram associação com a faixa etária (maior de 40 anos), entretanto, não se percebeu tendência de progressão conforme o aumento da idade. As barreiras percebidas para $\mathrm{AF}$ podem variar entre grupos de idade e de acordo com o contexto de vida das pessoas. Cenças entre grupos de idade e gênero podem ocorrer na frequência das barreiras relatadas, bem como nos domínios ou tipo de barreiras de AF. Por exemplo, os modelos socioecológicos consideram que múltiplos fatores podem influenciar a AF, incluindo variáveis intrapessoais, interpessoais e ambientai (Rech, Camargo, Araujo, Loch, \& Reis, 2018).

As barreiras ligadas a fatores comportamentais (mau humor, medo de lesionar-se, falta de interesse em praticar e preocupação com a aparência) são mais presentes entre os servidores analisados, podendo ser reflexo de quadros laborais estressantes que podem culminar em maiores índices de inatividade física e por consequência de desenvolvimento de doenças crônicas não transmissíveis (DCNT), altos índices de aposentadorias precoce e prevalência de excesso de peso com o avanço da idade (Siqueira, Reis, Souza, Pinho, \& Pinho, 2019), o que indica a necessidade de hospitais e centros de saúde instaurarem programas de incentivo à prática de atividade física para seus funcionários e colaboradores.

\section{Conclusão}

Os dados analisados permitem concluir que o baixo nível de atividade física pode ser considerado um fator associado a maiores queixas de limitações para a prática. Ressalta-se que barreiras como não gostar de AF, lesão e falta de companhia são mais frequentes em homens inativos, enquanto que a barreira medo de lesão apresentou maior proporção entre as mulheres ativas. 
Além disso, as barreiras preguiça, medo de sofrer lesão e falta de tempo livre demonstraram associação com a faixa etária, entretanto, não se percebeu tendência de progressão conforme o aumento da idade. Destaca-se no decorrer das faixas etárias as limitações para a prática de AF se alternam, porém, nunca deixam de existir.

Do ponto de vista prático, destaca-se a importância que gestores públicos promovam o desenvolvimento de programas de atividade física específicos para profissionais da saúde que trabalham em hospitais, considerando que muitas vezes a extensa carga horária de trabalho pode interferir na prática de atividade física.

\section{Referências}

Abreu, B. C., Abreu, G. C., Sampaio, V., \& Rodrigues, P. (2018). As barreiras percebidas para a prática de atividade física no lazer entre policiais militares. Revista Saúde Física \& Mental, 6(2), 31-42.

Alves, M. H., \& Pontelli, B. P. B. (2015). Doenças crônicas e a prática da atividade física no impacto das internações por causas sensíveis a atenção básica. Revista Fafibe On-Line, 8(1), 310-318.

Berria, J., Teixeira, D. M., Minatto, G., \& Petroski, E. L. (2016). Stages of Behavior Change for physical activity and associated factors among technical-administrative servers of a federal university. Revista Brasileira de Cineantropometria e Desempenho Humano, 18(4), 471-482.

Bittencourt, S. A., Santos, P. L., \& Oliveira, M. S. (2012). Motivação para mudança: análise fatorial da URICA para hábitos alimentares. Psico $U S F, 17(3)$.

Éric, G., \& Almeida, F. (2012). Nível de atividade física e barreiras percebidas para a prática de atividades físicas entre policiais militares. Revista Brasileira de Ciências do Esporte, 34(2), 433-448.

Frank, E., Dresner. Y., Shani, M., \& Vinker, S. (2013). The association between physicians' and patients' preventive health practices. Canadian Medical Association Journal, 185(8), 649-154.

Franklim, C., Daniele, B., Vitorino, C., Cieslak, F., \& Gregorio (2008). Nível de atividade física e suas possíveis barreiras em docentes universitários de Toledo-PR. Lecturas Educación Física y Deportes, 12(118).

Fransson, E. I., Heikkilä, K., Nyberg, S. T., Zins, M., Westerlund, H., Westerholm, P., Väänänen, A., \& et al. (2012). Job strain as a risk factor for leisure-time physical inactivity: An individual-participant meta-analysis of up to 170,000 men and women. American Journal of Epidemiology, 176(12), 1078-1089.

Gobbi, S., Sebastião, E., Papini, C. B., Nakamura, P. M., Valdanha Netto., A., Gobbi, L. T. B., et al. (2012). Physical Inactivity and Related Barriers: A Study in a Community Dwelling of Older Brazilians. Journal of Aging Research, 8.

Goulart, F. A. A. (2011). Doenças crônicas não transmissíveis: estratégias de controle e desafios e para os sistemas de saúde. Organização Pan-Americana da Saúde. < https://www.nescon.medicina.ufmg.br/biblioteca/imagem/4857.pdf>.

Hyeda, A., Costa, E. S. M., Sbardellotto, F., \& Ferreira, J. C. C. (2015). A aplicação da arquitetura de informação na gestão dos riscos das doenças crônicas em trabalhadores: uma análise preliminar. Revista Brasileira de Medicina do Trabalho, 14(1), 29-36.

Jesus, G. M., \& Jesus, E. F. A. (2011). Nível de atividade física e barreiras percebidas para a prática de atividades físicas entre policiais militares. Revista brasileira de ciências do esporte, 34(2), 433-448.

Justine, M., Azliyana, A., Vaharli, H., Zoolfaiz, S., \& Haidzir, M. (2013). Barriers to participation in physical activity and exercise among middle-aged and elderly individuals. Singapore Medical Journal, 54(10), 581-586.

Leão, J. M., Lisboa, L. C. V., Pereira, M. A., Lima, L. F., Lacerda, K. C., Ribeiro, M. A., \& Aguiar, E. A. S. (2015). Estágios motivacionais para mudança de comportamento em indivíduos que iniciam tratamento para perda de peso. Jornal Brasileiro de Psiquiatria, 64(2), 107-14.

Marcus, B. H., Banspach, S. W., Lefebvre, R. C., Rossi, J. S., Carleton, R. A., \& Abram, D. B (1992). Using the stages of change model to increase the adoption of physical activity among community participants. American Journal on Health Promotion, 6(6), 424-429.

Matsudo, S., Araújo, T., Matsudo, V., Andrade, D., Andrade, E., Oliveira, L. C. \& Braggion, G. (2012). Questionário Internacional de Atividade Física (Ipaq): Estudo de Validade e Reprodutibilidade no Brasil. Revista Brasileira de Atividade Física e Saúde, 6(2), 5-18.

Normansell, R., Smith, J., Victor, C., Cook, D. G., Kerry, S., Iliffe, S., \& et al. (2014). Numbers are not the whole story: a qualitative exploration of barriers and facilitators to increased physical activity in a primary care based walking intervention. BMC Public Health, 14:127. 
Research, Society and Development, v. 10, n. 12, e326101219588, 2021

(CC BY 4.0) | ISSN 2525-3409 | DOI: http://dx.doi.org/10.33448/rsd-v10i12.19588

Rech, C. R., Camargo, E. M., Araújo, P. A. B., Loch, M. R., \& Reis, R. S. (2018). Barreiras percebidas à atividade física de lazer na população brasileira. Revista Brasileira de Medicina do Esporte, 24(4), 303-309.

Siqueira, F. V., Reis, D. S., Souza, R. A. L., Pinho, S., \& Pinho, L. Excesso de peso e fatores associados entre profissionais de saúde da Estratégia Saúde da Família. Cadernos Saúde Coletiva, 27(2), 138-145.

Vieira, V. R., \& Vieira, J. V. (2019). Barreiras à prática de atividades físicas no lazer de brasileiros: revisão sistematizada. Pensar a Prática, 22 . 